\title{
Ruptured Aneurysm of Recurrent Artery of Heubner: A Case Report
}

\author{
Chang Eui Hong ${ }^{1}$, Yeongu Chung ${ }^{1}$, Yu Sam Won', Pil-Wook Chung ${ }^{2}$, Myung Ho Rho ${ }^{3}$ \\ Departments of ${ }^{1}$ Neurosurgery, ${ }^{2}$ Neurology, ${ }^{3}$ Radiology, Kangbuk Samsung Hospital, Sungkyunkwan University School of \\ Medicine, Seoul, Republic of Korea
}

Corresponding author:

Yu Sam Won

Department of Neurosurgery, Kangbuk Samsung Hospital, Sungkyunkwan University School of Medicine, 29, Saemunan-ro, Jongno-gu, Seoul 03181, Republic of Korea

Tel: +82-2-2001-2159

Fax: +82-2-2001-2157

E-mail: yusam.won@samsung.com

Received: August 4, 2019

Revised: August 19, 2019

Accepted: August 22, 2019
Recurrent artery of Heubner (RAH) aneurysms are extremely rare and few cases have been reported in the literature. A 53-year-old woman was transferred for evaluation and management of spontaneous subarachnoid hemorrhage (SAH). Cerebral angiography revealed a rupture of RAH aneurysm. The aneurysm was secured with a fenestrated right-angled Yasargil clip along the $\mathrm{RAH}$. Postoperative brain computed tomography showed a hypodense lesion at the left head of the caudate nucleus due to obliteration of the RAH. Fortunately, the patient experienced no neurologic deficits. To avoid iatrogenic obliteration of $\mathrm{RAH}$, intraoperative careful confirmation of its flow should be performed. We report a case of RAH aneurysm in a patient with $\mathrm{SAH}$.

Key Words: Aneurysm; Anterior cerebral artery; Subarachnoid hemorrhage

\section{INTRODUCTION}

Aneurysms of the lenticulostriate artery (LSA) are very uncommon; however, recurrent artery of Heubner (RAH) aneurysms of the LSA are very rare and only a few cases have been reported in the literature. Rupture of a LSA aneurysm can cause deep intracerebral hemorrhage or subarachnoid hemorrhage (SAH) according to location of aneurysms ${ }^{8}$. The natural history and etiology of RAH aneurysms are unknown. Only SAH caused by rupture of RAH aneurysms has been reported ${ }^{4,5,9)}$. Although various treatment methods (surgical clipping, endovascular treatment, and conservative treatment) can be considered, there is no consensus concerning a therapeutic strategy due to the rarity of cases. We report on a patient with SAH caused by rupture of RAH aneurysm.

\section{CASE REPORT}

A 53-year-old female presented to the emergency room of our hospital after the sudden onset of thunderclap headache, nausea, and lethargy. She did not have medical history of diabetes, hyperlipidemia, or hypertension. Her blood pressure at the emergency room was $117 / 69 \mathrm{mmHg}$ and other vital signs were stable. The patient appeared lethargic but answered all questions appropriately. Other neurological deficits were not noted. Brain computed tomographic (CT) (Fig. 1A) scanning showed diffuse SAH with a clot in the basal and both Sylvian cisterns. A CT angiogram was performed, which revealed an aneurysm of approximately $8 \mathrm{~mm}$ in the left proximal A1 segment of the anterior cerebral artery (ACA) (Fig. 1B-D). For the anatomic details of the surrounding vasculature, a cerebral angiogram was performed. A left internal cerebral angiogram revealed that the aneurysm originated from $\mathrm{RAH}$, which arose from the junction of $\mathrm{A} 1$ and the anterior communicating artery (ACoA), and its size was approximately $8 \mathrm{~mm}$ (Fig. 1E, F). There was no evidence of vasculitis, other aneurysms, or moyamoyalike-vessels, and all of the surrounding vasculature appeared normal. Because an endovascular embolization was thought difficult, we decided that microsurgical clipping would be more favorable.

The patient was taken to the operating room the following morning and positioned for a standard left pterional approach. After the craniotomy and dural opening, the Sylvian fissure was opened lateral to medial, and carotid and chiasmatic cisterns were opened in order. The A1 trunk was exposed thoroughly. At the junction of $\mathrm{A} 1$ and $\mathrm{A} 2$, the origin of $\mathrm{RAH}$ was visualized but an aneurysm was not seen. For further exposure, partial gyrus rectus corticotomy was performed; RAH aneurysm was visualized and appeared as a thrombus filled dilation (Fig. 2A). A fenestrated right-angled Yasargil clip was applied along RAH (Fig. 2B). After securing the aneurysm, the proximal and distal blood flow in RAH was confirmed using Doppler sonography. The distal blood flow of RAH decreased slightly after clipping but was maintained. After the confirmation, part of the aneurysm 

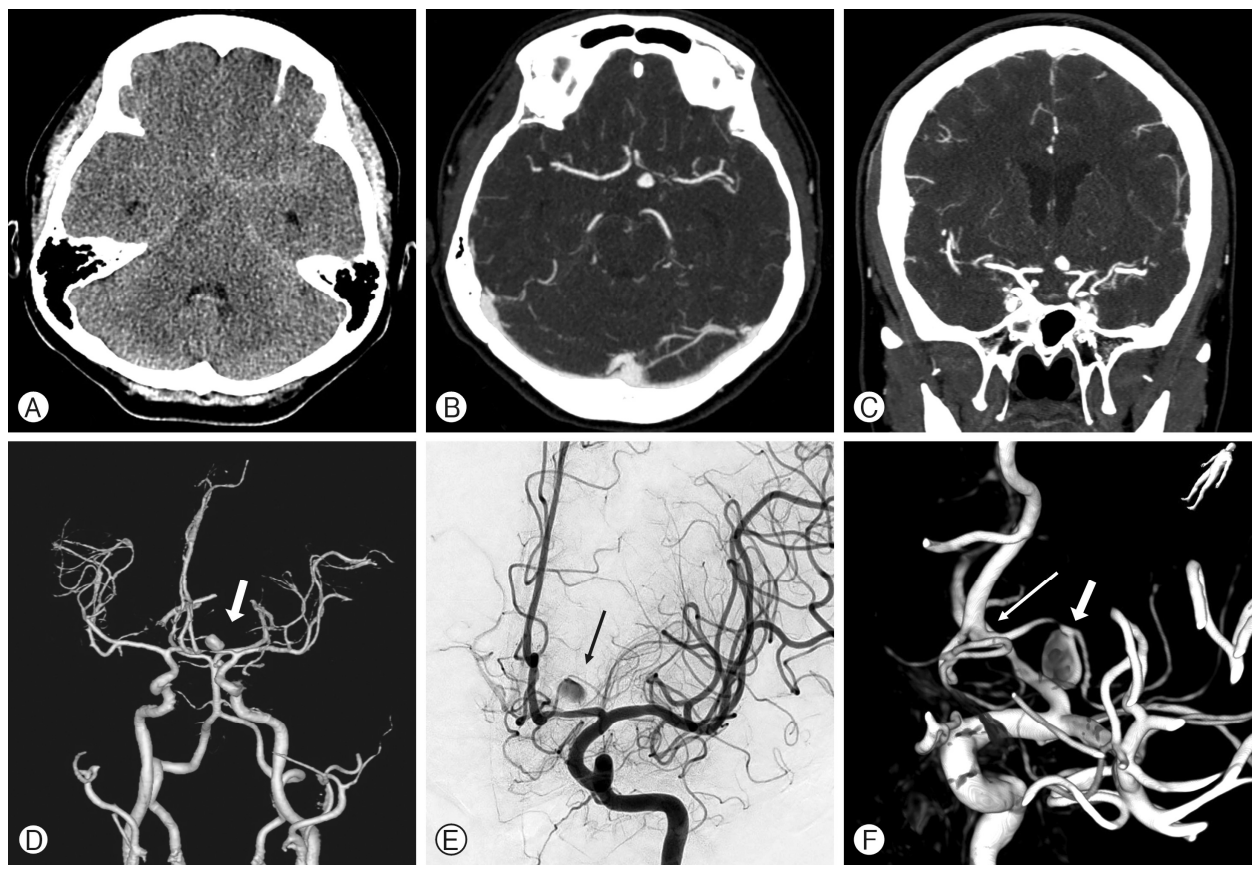

Fig. 1. (A) Brain computed tomography (CT) image reveals diffuse subarachnoid hemorrhage without intraventricular hemorrhage or intracerebral hemorrhage. The axial view $(B)$, coronal view $(C)$, and three-dimensional (3D) reconstruction image (D) of CT angiography show an aneurysm (arrow) arising from the proximal segment of the anterior cerebral artery ( $\mathrm{Al}$ portion). The oblique view (E) and $3 \mathrm{D}$ reconstruction image $(F)$ of angiography of a left internal cerebral demonstrate an aneurysm that originated from the recurrent artery of Heubner (thin arrow), which arose from the junction of $A 1$ and the anterior communicating artery (thick arrow); its size was approximately $8 \mathrm{~mm}$.
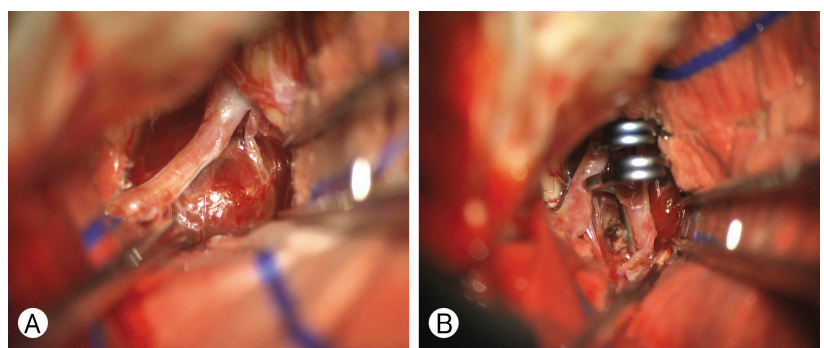

Fig. 2. Intraoperative view. (A) After partial gyrus rectus corticotomy, recurrent artery of Heubner aneurysm (RAH) was visualized. (B) A fenestrated right-angled Yasargil clip was applied along the RAH after securing the aneurysm.

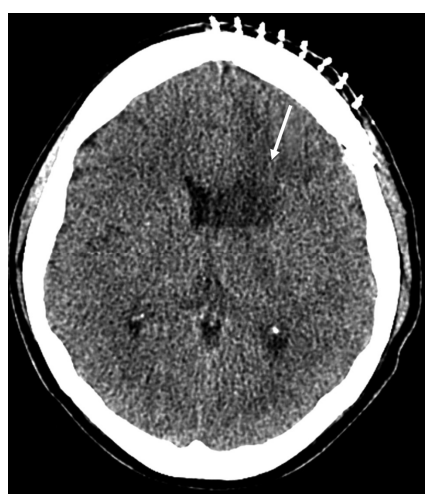

Fig. 3. Postoperative brain computed tomography image shows a hypodense lesion (arrow) at the left head of the caudate nucleus. was excised for a pathology. The cranium and incision were closed in a standard fashion.

The patient woke up with no focal neurologic deficit postoperatively, but the postoperative brain CT showed an area of hypodensity in the left caudate nucleus (Fig. 3). She was treated according to our standard protocol for SAH and monitored for vasospasm using transcranial Doppler sonography every other day. After 19 days, the patient was discharged from the hospital without neurologic deficits. The pathology report did not indicate possible infectious etiology.

\section{DISCUSSION}

The RAH is the largest vessel of the medial lenticulostriate arteries. The ACA is divided into the A1-precommunicating portion, A2 from the ACoA to the callosomarginal artery, and A3 distal to the callosomarginal artery. The RAH branches from A1 (3.6-14.3\%), from A2 (23.3-47.8\%), or at the junction of ACA-ACoA (43.4-62.3\%) ${ }^{1-3)}$. It runs parallel and posteriorly to $\mathrm{A} 1$ and penetrates the lateral portion of the anterior perforating substance ${ }^{6)}$. It supplies the anterior striatum (caudate nucleus and putamen), a portion of the globus pallidus, anterior hypothalamus, and anterior limb of the internal capsule ${ }^{1)}$. Its injury results in paresis of the contralateral upper extremity 
and face, aphasia (if damage occurs to the dominant hemisphere), and even memory loss or personality changes ${ }^{7}$. Injury also causes dysfunction of the tongue and palate. In many cases, occlusion of RAH leads to clinically silent infarctions that are limited to the head of the caudate nucleus and the neurological deficit tends to resolve over weeks to months ${ }^{1,4)}$.

Aneurysms arising from the proximal portion of ACA (A1: horizontal portion) are extremely rare and only a few cases of aneurysms arising from RAH have been reported ${ }^{9,10)}$. Vellore et al. ${ }^{9)}$ reported a case of RAH aneurysm in a patient with SAH, detected on delayed cerebral angiography. The patient demonstrated signs of photophobia and neck stiffness, but the first cerebral angiogram did not reveal any aneurysms. The following week, a second cerebral angiogram revealed a saccular aneurysm approximately $2.5 \mathrm{~mm}$ in size arising from the $\mathrm{RAH}^{9}$. Mansfield and Rahme ${ }^{4)}$ reported an exceptional case of ruptured dissecting aneurysm of the RAH in a patient with osteogenesis imperfecta. Ogata et al.") reported a case of a ruptured dissecting aneurysm of RAH, including the pathological findings.

Unfortunately, RAH aneurysms are rarely reported in the literature. We reviewed the literature of LSA aneurysms. Vargas et al. ${ }^{8)}$ classified LSA aneurysms into 3 types based on anatomical location. Type 1 describes aneurysms arising from the dorsal middle cerebral artery next to a perforating LSA but not incorporating the perforator. Type 2 is an LSA aneurysm from which the perforating artery or arteries arise (type 2A, type $2 \mathrm{~B}$ ). Type 3 describes a fusiform aneurysm beyond the first loop or turn of an LSA. Our case is most similar to type 3 according to the above classification but not the same. They reported type 3 aneurysms are the typical Charcot-Bouchard aneurysms associated with hypertension and associated with moyamoya-like vasculature.

\section{CONCLUSION}

We report the rare case of rupture of RAH aneurysm. RAH injury can result in contralateral hemiparesis with facio-brachiocrural predominance, epilepsy, athetosis, and cognitive and beha- vior disturbances. To avoid iatrogenic obliteration of RAH, intraoperative careful confirmation of its flow should be performed.

\section{CONFLICTS OF INTEREST}

No potential conflict of interest relevant to this article was reported.

\section{REFERENCES}

1. El Falougy H, Selmeciova P, Kubikova E, Haviarová Z: The variable origin of the recurrent artery of Heubner: an anatomical and morphometric study. Biomed Res Int 2013:873434, 2013

2. Izci Y, Seçkin H, Medow J, Turnquist C, Başkaya MK: Sulcal and gyral anatomy of the orbitofrontal cortex in relation to the recurrent artery of Heubner: an anatomical study. Surg Radiol Anat 31:439-445, 2009

3. Loukas M, Louis RG, Jr., Childs RS: Anatomical examination of the recurrent artery of Heubner. Clin Anat 19:25-31, 2006

4. Mansfield K, Rahme R: Dissecting aneurysm of the recurrent artery of Heubner in a patient with osteogenesis imperfecta. Can J Neurol Sci 42:461-465, 2015

5. Ogata A, Sakata S, Okamoto H, Abe T: Ruptured dissecting aneurysm of the recurrent artery of Heubner: Consideration of pathological findings. Neurol India 65:623-625, 2017

6. Pai SB, Kulkarni RN, Varma RG: Microsurgical anatomy of the anterior cerebral artery - anterior communicating artery complex: An Indian study. Neurol Asia 10:21-28, 2015

7. Rhoton AL, Jr.: The supratentorial arteries. Neurosurgery 51: S53-S120, 2002

8. Vargas J, Walsh K, Turner R, Chaudry I, Turk A, Spiotta A: Lenticulostriate aneurysms: a case series and review of the literature. J Neurointerv Surg 7:194-201, 2015

9. Vellore Y, Madan A, Hwang PY: Recurrent artery of Heubner aneurysm. Asian J Neurosurg 9:244, 2014

10. Wanibuchi M, Kurokawa Y, Ishiguro M, Fujishige M, Inaba K: Characteristics of aneurysms arising from the horizontal portion of the anterior cerebral artery. Surg Neurol 55:148-154, 2001 